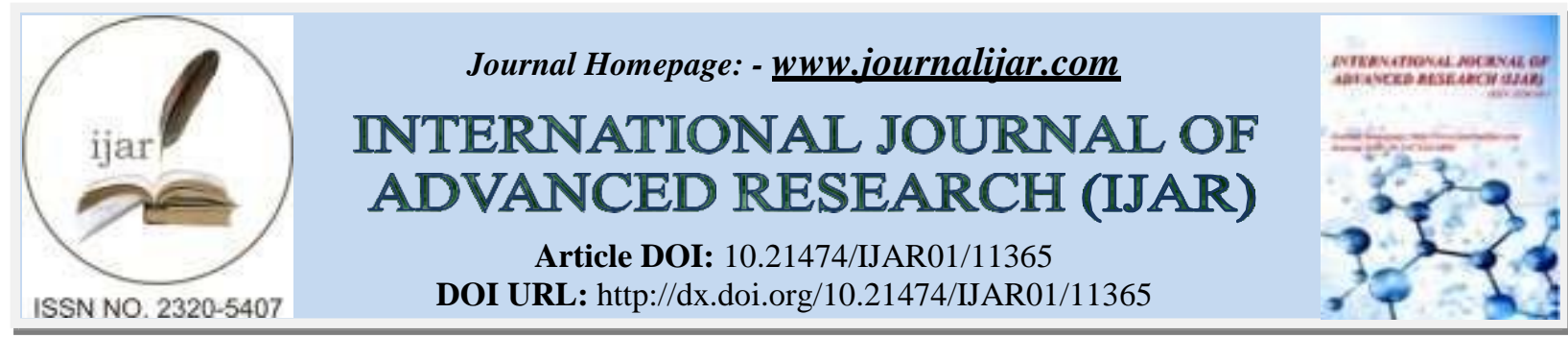

RESEARCH ARTICLE

\title{
NEW MODERN NANOENERGETICS OF THE SIXTH TECHNOLOGICAL REVOLUTION
}

Manuscript Info
Manuscript History
Feceived: 20 May 2020
Published: July 2020
Key words:-
Non-Ideal Quantum Plasma, Modern
Energy, Energy Release Mechanism,
New State Of Matter - Quantum Plasma
Condensate, Alternative, Efficient,
Renewable, And Stable Fundamentally
New Source Of Energy Source, Plasma
Combustion Of Fuels, Purification Of
The Planet's Ecosphere, UMoID/A

A.V. Kulakov

rofessor, Institute of Functional Economic Systems, Moscow, Russia.

\begin{abstract}
A fundamentally new direction in theory and practice of quantum nonlinear phenomena in a non-ideal and very close to degenerate plasma has been developed. For the first time, the existence of a new state of matter-imperfect plasma-quantum plasma condensate combining the properties of ordinary liquid and ionized plasma has been theoretically predicted and confirmed. The analysis of modern nanoenergetics has been conducted, special attention has been paid to its ecological state, the quantum non-ideality of a non-degenerate plasma and the exchange interaction of electrons in it, which leads to the efficient energy release in the plasma process being studied, have been investigated. The discovery of this kind of phase state of a non-ideal non-degenerate plasma and its use in engineering and technology allows creating a fundamentally new effective, renewable, cheap, and stable nanoenergetics on a global basis. This energy source is the only environmentally friendly source that does not destroy and pollute our planet, while its use purifies our ecosphere. Based on the author's pioneering discoveries in the qualitative research of nonlinear quantum phenomena in a non-ideal and close to non-degenerate plasma and the proposal of a fundamentally new, effective, and harmless energy source, the use of which purifies all spheres of the planet Earth, and also his proposals of fundamentally new devices - Universal Modules of Industrial Disintegrators/Activators- the possibility of implementing ambitious, efficient, and self- sustaining state projects based on the developed nanotechnologies is considered. During the research of nonlinear quantum phenomena in non-ideal and close to degenerate one, the author has developed and offered solutions to the triumvirate of problems that allow: create a modern, efficient, safe, harmless, and renewable nanoenergetics that meets the actual challenges; reduce the threat of the coronavirus pandemic; mitigate the effects of crises and deal with the global economic collapse. The work shows that the use of currently existing energy imposes a health hazard, reduces people's immunity, and (naturally) resistance to the coronavirus. Measures are proposed to minimize the consequences of the coronavirus pandemic on Earth. Information on the proposed technical projects and Universal Modules of Industrial Disintegrators/Dispersants, which allows to introduce the new developed nanotechnologies of the new perfect and cheap materials and products and create millions of jobs, is provided.
\end{abstract}

Copy Right, IJAR, 2020,. All rights reserved. 


\section{Introduction:-}

The situation of our planet Earth is very alarming. Humanity is moving towards the new Sixth Technological Revolution, which, like in every transition, could lead to a change in ownership of assets and financial flows and, possibly, reformat the entire system of activities on our planet (in extremis, including social unrest). We do not know what the oncoming economic crisis will be like, the percentage of GDP losses in the U.S. and the European Union, and the consequences of these losses.

The World Health Organization cannot predict the consequences of the spread of coronavirus (COVID-19), the causative agent of which was the novel coronavirus SARS- Cov-2.

Doctors are concerned about some of the features of this coronavirus, such as multivariance of its symptoms, inability to establish an "intermediate carrier" of the virus that could infect a person, virus mutation, inability to quickly obtain a vaccine against coronavirus, the possibility of animals' bodies serving as "fomites," or surfaces that virus particles can land on and be transmitted by touch, and a large number of infected people.

This situation requires immediate antiviral and energy measures.

This article, based on the results of fifty years of work on development of breakthrough energy technologies in theory and practice of a non-ideal, close to non- degenerate plasma, started by $\mathrm{PhD}$ in Physics \& Mathematics A.A. Rumiantsev who, unfortunately, passed away in 1990. He predicted and confirmed the existence of a fundamentally new state of physical matter - quantum plasma nanocondensate - liquefied plasma, combining the properties inherent in standard liquid and ionized plasma - a fundamentally new, effective, and harmless energy source, the use of which helps reduce the pollution caused by waste. Purification of planet Earth, which has been contaminated to the limit, in some areas of which gas contamination, slagging, and municipal waste exceed all permissible norms, will lead to a boost in the immune systemof the population, which will make it possible for humans to exist safely in the presence of viruses.

Naturally, The aim of this work is to develop solutions to three global problems that determine the possibility of a satisfactory existence of people on planet Earth:

1. What should be the modern energetics;

2. Hazard reduction - Coronavirus pandemic control;

3. Mitigating the effects of the current economic crisis and dealing with the global economic collapse.

Methodology and materials concerning the problem A - What should be the modern energetics:

The author of this article was the first to discover that when plasma is densified even before the onset of the degeneration stage, the overlap of electron wave clouds becomes significant. Overlapping effects create the first-order effect in relation to de Broglie wavelength and interionic distance. If in substances being in the usual phase state, the overlap also exists, but with increasing distances between atoms decreases exponentially, then in a plasma, since the spectrum of quantum energy states of electrons in it is continuous, the effect of shells overlaps decrease with increasing interparticle distances slows down significantly and described by a power law dependence [1].

As a result, even in a plasma of moderate density with $\sim 10^{20-3}$ ion concentration, such a picture of particle interconstrictions arises that corresponds to a chain of successively overlapping electron clouds, and each of the chain branches extends for a distance of the screening radius order. The whole chain covers the entire plasma as a whole plasma ions "captured" by this chain are attracted to each other, a phase plasma transformation occurs.

The transition to a new state is accompanied by the energy release equal to the heat of transformation. The quantitative analysis below shows that the energy release can be very significant and exceed the corresponding specific energy release during the standard fuel combustion [1], [2]\{3|4\}\{5|6\}[7][8]

The fact that the overlap of electron shells of atoms leads to their effective coupling is well known from the theory of chemical bonding. It is enough, for example, to point out the Heitler-London theory of molecular forces, in which such forces are detected when calculating the simplest molecules based on the application of the variational method. And now, this method is most often used to explain and calculate the structure of molecules and the forces acting between 
the atoms composing them. Variational methods in physics belong to the category of intuitive, posterior. A consistent heuristic theory can only be a theory that is based on a direct solution to the fundamental equation of quantum theory the Schrodinger equation. Such a theory is the perturbation theory considering exchange forces (or the Pauli Exclusion Principle which the author used to solve the Schrodinger equation [1], [2]

Extended to the class of states of the continuous spectrum, which is realized with respect to the states of electrons in a plasma, this theory allows explaining the already observed features of the plasma phase, as well as to predict and use those phase properties that can and should be used by modern engineering and technology. We are talking about the manifestations of plasma non-ideality because the energy of theCoulomb interaction in such a plasma is comparable with the thermal background energy, and the non-ideality of quantum origin.

The results of a rigorous solution to the Schrodinger equation, lass of continuous spectrum states, in [1], [2] showed that quantum effects were significant in case of the so- called non-degenerate plasma, when the average interelectronic distance exceeds the de Broglie wavelength of thermal electrons several times, that is, when the following inequality is made

$$
\lambda=\frac{h}{p} \geq\left(\frac{1}{5}-\frac{1}{10}\right)(\mathrm{zn})^{-1 / 3} \text {, }
$$

where $\chi$ is a de Broglie wavelength of thermal electrons, ( ) $1 / 3-r$ is anaverage-

electron distance. The exchange interaction of electrons under such conditions leads to the fact that ions attract each other; the binding energy of the latter becomesnegative.

A phase transition occurs in substances, accompanied by the energy release, which, however turns out to be significantly larger than under the standard phaseprocesses.

The specific energy release (per gram) corresponding to the latent heat of phase transformation is as follows [1]: $\mathrm{E}_{0}=10 \mathrm{z}^{3} \mathrm{e}^{2} \mathrm{n}^{1 / 3}$ /

Where $\mathrm{e}=14.8 \cdot 10^{-10} \mathrm{cgs}$ is an electron charge, $\mathrm{z}$ is a degree of atoms ionization, $\mathrm{m}_{\mathrm{i}}$ is an ion mass.

Assuming $\mathrm{n}=10^{21} \mathrm{~cm}-3$, to evaluate $\mathrm{z}=2,=2 \cdot 10^{-23} \Gamma$, we obtain ${ }_{0}=10^{13} \mathrm{erg} / \mathrm{g}=1 \mathrm{BOD} / \mathrm{g}$, which exceeds the energy release of the most efficient fuels, with the exception of nuclear materials.

Quantumcondensate of a non-ideal plasma is the only renewable, harmless, efficient, cheap, reproducible, and stable source of energy on planet Earth. It allows to consider it as corresponding to the Sixth Technological Revolution, and its use as an industry for the processing and disposal (recycling) of waste created on planet Earth.

The author of this article has developed methods for converting the quantum plasma condensate energy into thermal [8] and electric energy [7], ultraviolet and X-ray radiation [4], energy of generation and acceleration of charged particles [6].

Materials and methods concerning the problem B - Hazard reduction - Coronavirus pandemic control: Scientists believe that the population of the Earth will die not from nuclear weapons but from waste. 95-97\%\% of what is extracted from the earth converts either into an intermediate state or is buried. The world is in a state where the environmental damage of technology has exceeded the capacity of our planet and people living on it.

As a result of its vital activity, mankind has accumulated and placed a prohibitive amount of industrial and domestic waste on the Earth's surface.

Waste is disposed of and continues to be disposed of in dumps, storages, disposal sites, and at special landfills that occupy vast areas since the annual level of waste processing does not exceed 5-7\%.

At the same time, the process of extracting various minerals from the earth's crust, which are necessary for accelerated economic growth and meeting the demands of "consumer society": ores, energy raw materials, and mineral components required for growing production, is intensifying. 
As a result, the surface of our planet is covered with new dumps, slagheaps, sludge depositories, abandoned quarries, etc.

All these factors negatively affect the environment and the people health, steadily making the disabled community of people with a range of concomitant diseases and low immunity grow. Intensive development of the old economy and the concept of "consumer society" will accelerate the accumulation of waste in the 21 st century.

Tables No. 1, 2 show the compositions of typical products and wastes of various industrial productions that are subject to worldwide subsequent high-temperature disposal — burning with flue-gas emission.

Table 1:- Compositions of oxidized steelmaking slag, cement clinker, and portland cement of CEM 1 grade

\begin{tabular}{|c|c|c|c|c|c|c|c|c|}
\hline \multirow[b]{2}{*}{ Name } & \multicolumn{8}{|c|}{ Content, \% } \\
\hline & $\mathrm{CaO}$ & & $\mathrm{Si}$ & $\mathrm{MgO}$ & & $\mathrm{MnO}$ & $\mathrm{Fe}$, prills & S \\
\hline $\begin{array}{l}\text { Oxidized steelmaking } \\
\text { slag }\end{array}$ & $40-55$ & $1.5-3$ & $15-19$ & $1.5-2.5$ & $18-25$ & $4-7$ & $4-6$ & - \\
\hline $\begin{array}{l}\text { Remelted and partially } \\
\text { reduced slag }\end{array}$ & $61.7-63$ & $1.8-3.7$ & $18-24$ & $1.8-3.1$ & $4.5-5.2$ & $2.5-4$ & 0 & - \\
\hline Standard cement clinker & $60-67$ & $3-8$ & $17-25$ & $2.5-5$ & $4-5$ & 一 & 0 & 一 \\
\hline $\begin{array}{l}\text { Typical CEM } 1 \text { portland } \\
\text { cement }\end{array}$ & $62-64$ & 5.5 & 21.5 & 1.5 & $3-4$ & - & 0 & 1.9 \\
\hline
\end{tabular}

Red bauxite slug is a fine substance of the following chemical composition, \%

\begin{tabular}{|l|l|l|l|l|l|l|l|l|}
\hline & $\mathrm{CaO}$ & $\mathrm{Si}$ & & $\mathrm{MgO}$ & $\mathrm{Ti}$ & $\mathrm{S}$ & $\mathrm{O}$ \\
\hline $40-55$ & $8-11$ & $5-15$ & $14-16$ & $0.5-1.4$ & $2-5$ & up to 2 & $0.2-0.5$ & up to 2 \\
\hline
\end{tabular}

Table 2:- Average slag compositions of non-ferrous metallurgy and heat power enterprises.

\begin{tabular}{|c|c|c|c|c|c|c|c|c|c|c|c|c|}
\hline \multirow[b]{2}{*}{ Name } & \multicolumn{11}{|c|}{ Content, $\%$} & \multirow{2}{*}{$\begin{array}{l}\text { Melting } \\
\text { temperature } \\
\text { OC }\end{array}$} \\
\hline & $\mathrm{Si}$ & $\mathrm{FeO}$ & $\mathrm{CaO}$ & & $\mathrm{MgO}$ & $\mathrm{Cu}$ & Co & $\mathrm{Ni}$ & $\mathrm{Zn}$ & $\mathrm{Pb}$ & $\mathrm{S}$ & \\
\hline $\begin{array}{l}\text { copper } \\
\text { smelter slags }\end{array}$ & $\begin{array}{l}32- \\
45\end{array}$ & $\begin{array}{l}25- \\
45\end{array}$ & 12 & $\begin{array}{l}3.2- \\
9.7\end{array}$ & $2-11$ & $\begin{array}{l}0.3- \\
0.9\end{array}$ & - & - & $0.5-1$ & $\begin{array}{l}0.22- \\
0.8\end{array}$ & $\begin{array}{l}0.4- \\
1.2\end{array}$ & $1100-1150$ \\
\hline $\begin{array}{l}\text { nickel shaft } \\
\text { furnace slags }\end{array}$ & $\begin{array}{l}39- \\
45\end{array}$ & $\begin{array}{l}16- \\
24\end{array}$ & $\begin{array}{l}12- \\
21\end{array}$ & $\begin{array}{l}4.5- \\
7.5\end{array}$ & $9-17$ & - & $\begin{array}{l}0.010- \\
0.024\end{array}$ & $\begin{array}{l}0.1- \\
0.17\end{array}$ & - & - & $\begin{array}{l}0.43- \\
0.5\end{array}$ & $1100-1200$ \\
\hline $\begin{array}{l}\text { nickel } \\
\text { converter } \\
\text { slags }\end{array}$ & $\begin{array}{l}25- \\
35\end{array}$ & $\begin{array}{l}40- \\
60\end{array}$ & $2-3$ & $3-10$ & $2-4$ & $\begin{array}{l}0.1- \\
0.2\end{array}$ & $\begin{array}{l}0.01- \\
0.02\end{array}$ & $\begin{array}{l}0.3- \\
0.7\end{array}$ & - & - & $\begin{array}{l}2-3[ \\
]\end{array}$ & $1100-1200$ \\
\hline \multirow[t]{2}{*}{$\begin{array}{l}\text { lignite fired } \\
\text { heat power } \\
\text { plants ash }\end{array}$} & $\begin{array}{l}54- \\
55\end{array}$ & $\begin{array}{l}2.5- \\
10\end{array}$ & $\begin{array}{l}1.6- \\
2.5\end{array}$ & $\begin{array}{l}24.7- \\
25.2\end{array}$ & $\begin{array}{l}2.5- \\
2.6\end{array}$ & - & - & - & - & - & $\begin{array}{l}0.1- \\
0.3\end{array}$ & 1400 \\
\hline & $\begin{array}{l}44- \\
49\end{array}$ & $\begin{array}{l}7- \\
20\end{array}$ & $6-16$ & $9-20$ & $5-13$ & - & - & - & - & - & - & $1300-1350$ \\
\hline
\end{tabular}


A scientist from Martin Luther University Halle-Wittenberg Ogen identified the relationship between air pollution by nitrogen dioxide and the mortality rate due to a novel coronavirus by publishing his study on the Science Direct platform.

Ogen's analysis shows that of 4,443 deaths caused by coronavirus in 66 administrative regions in Italy, Spain, France, and Germany $78 \%$ are concentrated in only five regions where the air is most polluted, four of them are in Northern Italy and one in the autonomous community of Madrid.

It appears that only the difference in air pollution can explain the difference in mortality from coronavirus in China compared to Vietnam, Laos, Cambodia; in the USA compared to Mexico and Canada and in England compared to Turkey.

The today's situation with coronavirus in the USA gives rise to a concern.

At the same time, it is necessary to use, in the literal sense of the word, waste that destroys the planet's population: industrial waste, sludge depositories, mining dumps, and landfills as a material for obtaining plasma quantum condensate that is extremely useful for humanity as fuel which would destroy waste and purify three spheres of our planet.

There is only one principal rescue from viruses on our planet - an immediate transition to the new nanoenergetics $\langle 7|8| 4\rangle$, saving humanity from destruction.

There is no doubt that this is exactly what (use of waste, "revaluation of values," even the struggle for waste) will be the characteristic feature of civilization of the 21st century. Machinery, like nature, must be rational.

Thus, we have developed a fundamentally new direction in theory and technology of a non-ideal plasma. The research made it possible to predict and then experimentally discover the existence of a fundamentally new state of matter - a non-ideal quantum plasma - quantum plasma nanocondensate which combines the features typical of an ordinary liquid (fluidity, surface tension, internal correlations) and the signs typical of an ionized plasma in the usual sense. The theory developed in our research has identified those applications of quantum plasma condensate that should immediately be used by modern machinery and technology, as well as extremely mitigate the effects on people of any strains of coronaviruses.

Materials and methods concerning the problem C - Mitigating the effects of the current economic crisis and dealing with the global economic collapse.

This part of the article was completed jointly with Candidate of Sciences in Physics and Mathematics V.A. Rantsev Kartinov

The versatility and high efficiency of the Universal Modules of Industrial Disintegrators/Activators (UMoID/A [[9]], [10] (proposed and constructed by A.V. Kulakov and V.A. Rantsev-Kartinov) is preconditioned by the multitude and activity of the processes occurring in its working area:

1. unique ability to grind substances to units of $\mathrm{nm}$;

2. crushing by the "constrained blow";

3. electromagnetic erosion;

4. plasma exposure;

5. ultrasound (when processed in a liquid medium);

high energy density of magnetic induction in its working area (with $\mathrm{B} \sim 10^{4} \mathrm{Gs}, \mathrm{W} \sim 0.4 \mathrm{~J} / \mathrm{cm}^{3}=410^{5} \mathrm{~J} / \mathrm{m}^{3}$ ), Bmagnetic field strength, W- magnetic induction energy density is hundreds of times higher than the energy density in the working areas ofother similar devices;

1. almost one hundred percent efficiency;

2. low material consumption.

The UMoID/A design is schematically illustrated in Fig. 1. 

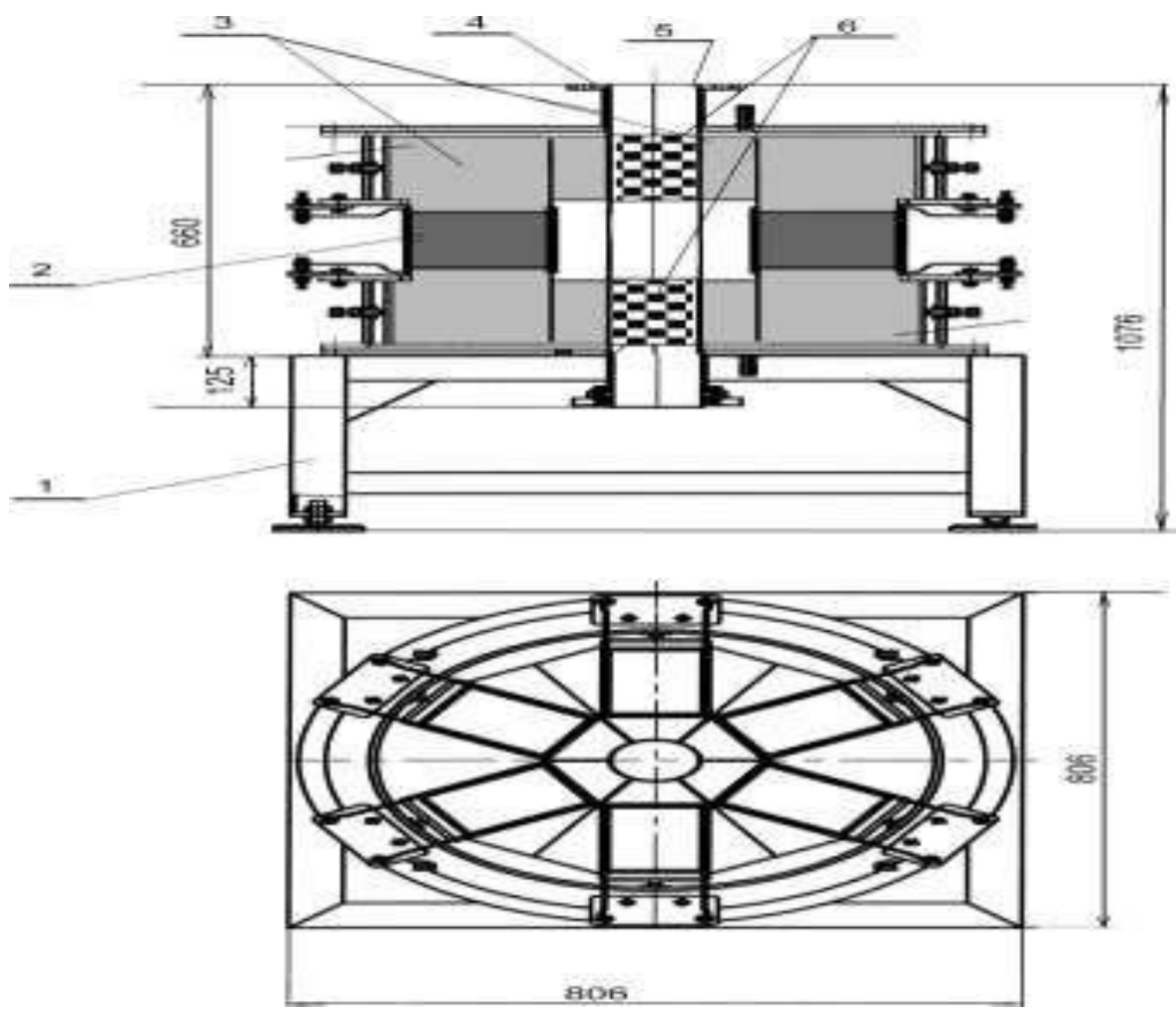

Fig 1: A schematic diagram of the UMoID/A installation:

1. support, (X18H9T); 2- magnetic coils;

2. inductor; 4 - main pipe;

Sleeve (X18H9T) with working areas; 6 - working areas with a workingmedium.

A schematic diagram of a continuous industrial grinding workshop is illustrated in Fig. 2. Figure 3 illustrates a schematic sketch of a prototype model installation for the production of materials on an industrial UMM-P scale. The support of this device should allow changing the slope of this module, providing adjustment of the processed material passage speed through the module. Schematically, this device represents a serial joining of separate modules with two working areas also patented by the authors.

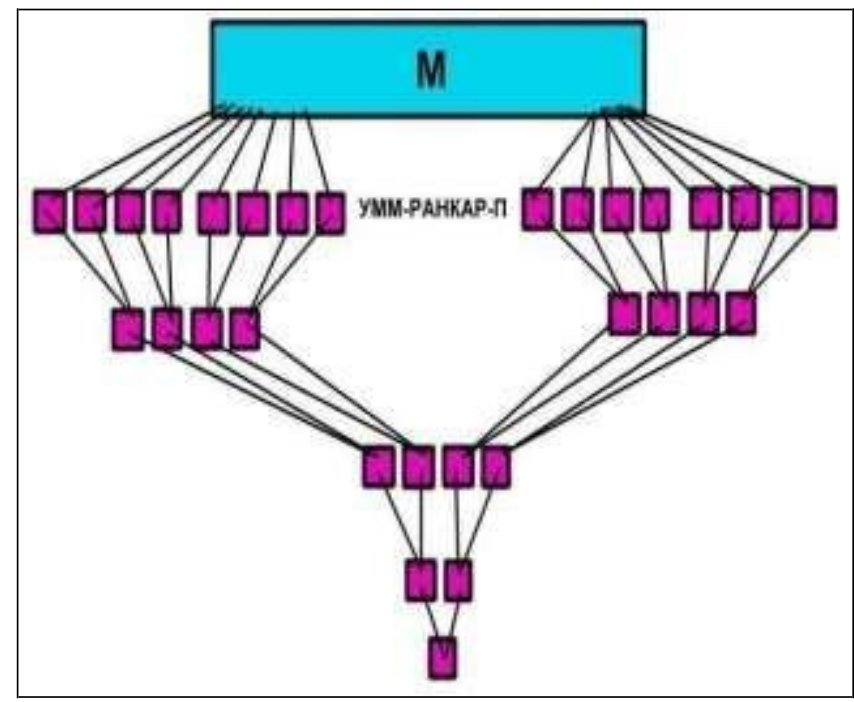

Fig 2:- The schematic diagram of a continuous industrial grinding workshop: $\mathrm{M}$ - material pre-grinding mill $\mathrm{UMoID} / \mathrm{A}$ - material continuous industrial grinding modules. 


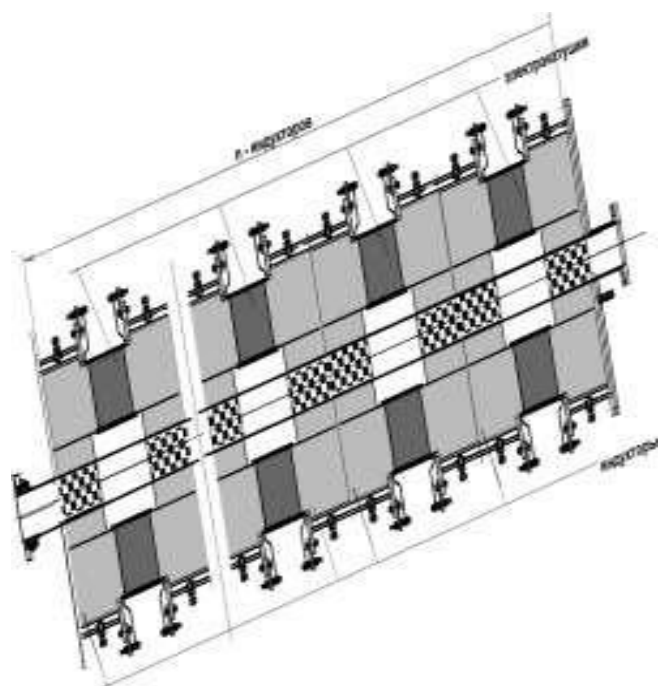

Fig. 3:- The schematic sketch of the UMoID/A prototype model installation.

Understanding clearly the novelty, complexity, and originality of the problems considered in the article, as well as the lack of predecessors in theory and practice of the energetics of a non-ideal nanoplasma - quantum plasma condensate, the authors of this section of this article realized that it was necessary to create a Laboratory NanoModule LNM device with a vortex ferromagnetic layer with their last ounce of strength and to conduct a series of numerous laboratory studies the results of which can confirm the validity of the theory of non-ideal plasma quantum condensate stated by the author in the article, where it is necessary to follow the dictum of the famous Russian scientist academician P.L. Kapitsa "Experience is the only evidence that is convincing for everyone" [11].

\section{The LNM provides:}

convenience of laboratory testing precisely in the fractional mode of new NT in various areas of industrial production; an increase in efficiency of mixing and grinding various media by increasing the magnetic field energy density in its working zone (WZ) both by providing the passage of the module magnetic flow through only one WZ and by doubling the ratio of the WZ length to its diameter, significantly reducing the marginal diffused flow of the magnetic field induction; simplicity of its repair and operational maintenance. Fig. 4 illustrates a schematic diagram of the LNM,

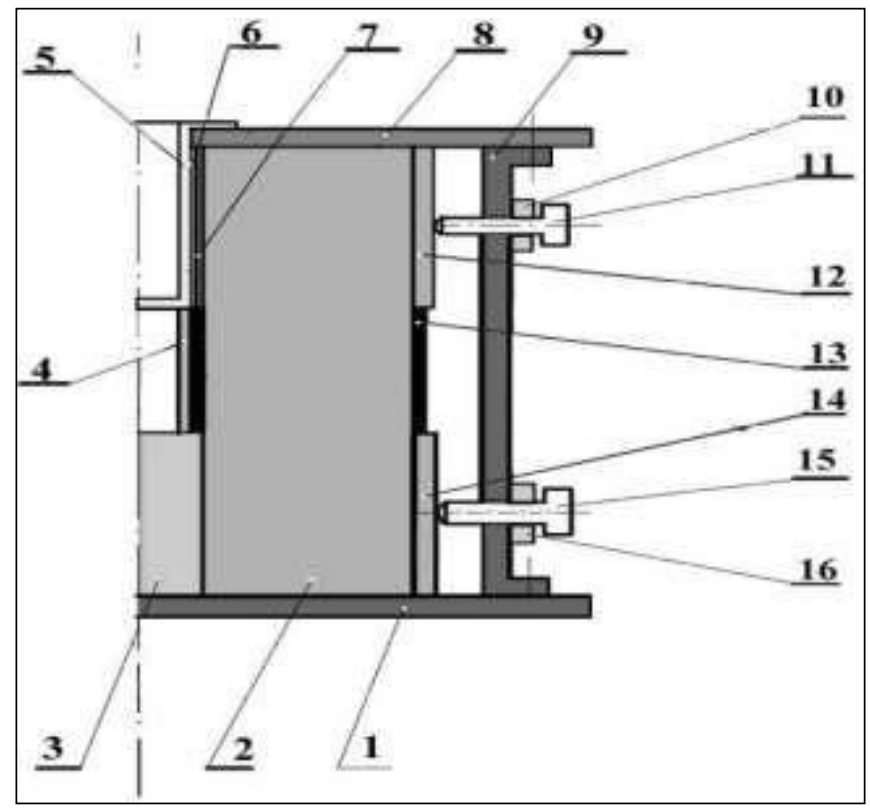

Fig 4:- The schematic diagram of the developed LNM: 
1. LNM bottom plate;

2. matrix supports of the LNM magnetic inductor 6 sections;

3. hexagon, shorting the inductor magnetic flows;

4. sleeve;

5. cylinder, limiting the volume of the LNM WZ;

6. WZ adjusting cylinder;

7. poles of the LNM electromagnetic inductor;

8. LNM top plate;

9. mounting and adjusting brackets of the inductor;

10. lock nuts of the inductor sections pole area mounting and adjusting screws;

11. mounting and adjusting screws of the inductor sections pole area;

12. plates for mounting and adjusting screws of the inductor sections pole area;

13. electromagnetic inductor coils;

14. plates for mounting and adjusting screws of the inductor sections lower area;

15. mounting and adjusting screws of the LNM inductor sections lower area;

16. lock nuts of the LNM inductor sections lower area mounting and adjusting screws.

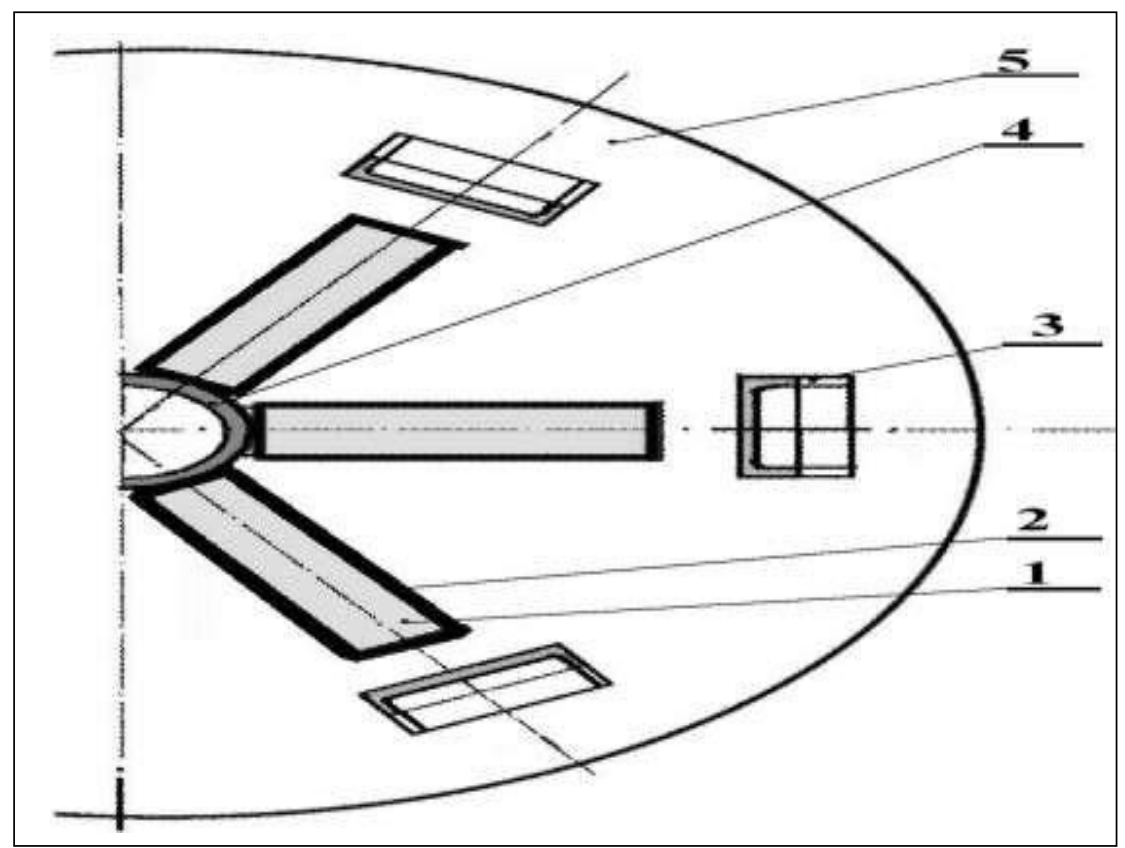

Fig 5:- Top view of the LNM along the transverse center section in Fig. 3.

1. LNM magnetic inductor 6 sections matrix supports;

2. electromagnetic inductor coils;

3. mounting and adjusting brackets of the inductor;

4. sleeve;

5. LNM bottom plate.

The scopes of UMoID/A and LNM industrial application can be:

1. construction industry, especially ocean construction;

2. oil producing and oil refining industry;

3. chemical industry;

4. paint and varnish industry;

5. agricultural industry (for example, cheap animal feed);

6. perfumery industry;

7. ecology.

spent a huge amount of time, effort, and personal resources on LNM experimental research in difficult conditions and new nanotechnologies development and experimental development. 
A preliminary assessment of the authors shows that the use of these units in real technologies can yield a profit of $(0.3 \div 30) \cdot 10^{6} € / \mathrm{g}$ per 1 module! The payback in this case for various technologies can be $(7 \div 300)$ days. So, for example, the modules themselves pay back their cost in just 70 hours of continuous operation in almost any technology when operating these units. Usually, they consume only 20\% of their rated electrical power from the network, which must be taken into account when calculating their operating efficiency in technology.

During the experiments, the following particular results were obtained:

when melting a mixture of quartz sand with alkalis, soda, or potash, the so-called "liquid glass" (LG) or alkaline salts of metasilicic acid are obtained. Metasilicic acid itself (MA) is obtained under the influence of a dilute solution of a strong acid, for example, hydrochloric acid on LG:

\section{$\mathrm{Na} 2 \mathrm{SiO} 32 \mathrm{HCl} 2 \llbracket \mathrm{NaCl} \mathrm{H} 2 \mathrm{SiO} 3$. (7)}

MA is activated by calcium oxides when they are jointly mixed together in a binder. In this case, linear arrays of silicon ions, interconnected according to the following scheme are connected:

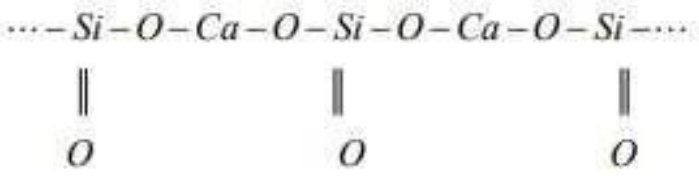

This leads to the fact that there is a significant difference in the binder compression and bending strength (tension). The effect of a more concentrated solution of hydrochloric acid on liquid glass leads to the ortho-silicic acid (OCA) formation according to the reaction given below:

2Na2SiO3 $\square 4 \mathrm{HCl} \square 4 \square \mathrm{NaCl} \square \mathrm{H} 4 \mathrm{SiO} 4 \square \mathrm{SiO} 2$,

The valence-bond structure of silicon in this acid is similar to the valence-bond structure of carbon in diamond. This condition leads to an increase and some isotropy of the strength of the binder obtained, the spatial structure of which, when activated by calcium and hydration, represents the three-dimensional fractal structure of calcium ions on tetravalent centers of silicon ions.

The invention belongs to a new industrial method ofobtaining the ortho-silicic acid which has a diamond-like structure of silicon valence bonds by directly dissolving the modified (by grinding silica sand to fineness of $\sim 10 \mathrm{~nm}$ in water during the preparation and activation of a homogeneous aqueous nano-suspension from it which can be achieved by means of the following patented nanotechnology using the Universal Module of Industrial Disintegrators/Activators (UMoID/A), (ME) Eurasian patented № 201800299 by the authors:

(The experiment was conducted using one of the LNM samples available to us)

at the first stage of operation (with the working medium elements (WME) in the form of cylinders with a diameter of $\sim 2 \mathrm{~mm}(0.08 \mathrm{in})$ and length of $\sim 10 \mathrm{~mm}(0.4 \mathrm{in})$ from hardened magnetically hard steel or balls with a diameter of $\sim 5 \mathrm{~mm}(0.2 \mathrm{in})$ from ball bearings) it is necessary to grind the original quartz sand to a fineness of $\leq 50 \mu \mathrm{m}$;

at the second stage (with WME in the form of cylinders with a diameter of $\sim 1 \mathrm{~mm}(0.04 \mathrm{in})$ and length of $\sim 10 \mathrm{~mm}$ (0.4 in) from hardened magnetically hard steel) finish grinding of the obtained quartz powder (KP) to fineness of

$\sim 10 \mathrm{~nm}$, thereby completing the process of modifying the KP, i.e., to obtain it in the form nanopowder (NP) of modified quartz sand (MQS) with a fineness of $\sim 10 \mathrm{~nm}$; at the third stage, we add water with the temperature from 1 to $30^{\circ} \mathrm{C}\left(32\right.$ to $\left.86^{\circ} \mathrm{F}\right)$ to the nano-powder and, as before, activating this mixture, we obtain a nonideal plasma with an ion concentration of $\mathrm{n} \sim 10^{20} \mathrm{~cm}^{-3}$ and a particle constriction occurs to which a chain of successively overlapping electron clouds corresponds, at that each branch of the chain extending over a distance of the screening radius order. 
The whole chain covers the entire plasma as a whole — plasma ions, "captured" by this chain are attracted to one another, a phase transformation of the plasma occurs. The transition to a new condition is accompanied by the energy release equal to the transformation heat.

The process of energy release is accompanied by a phase transformation of a new type of substance. First, a plasma discharge forms a substance such as a plasma liquid and then, upon further cooling, solid conglomerate-crystalline formations are formed. We can find such transformations, for example, in nature during volcanic eruptions. It seems that the solid plasma phase is a substance with some new unpredictable properties. In any case, during the "combustion" as a result we get new, maybe very useful, materials. This area can be called electric discharge plasma metallurgy.

OSA has found industrial application in many sectors of industrial production, but we are particularly interested in the possibility of using it in the creation and production of particularly strong building bonding materials from various local slags, volcanic/rock formations, composites with unique structural properties.

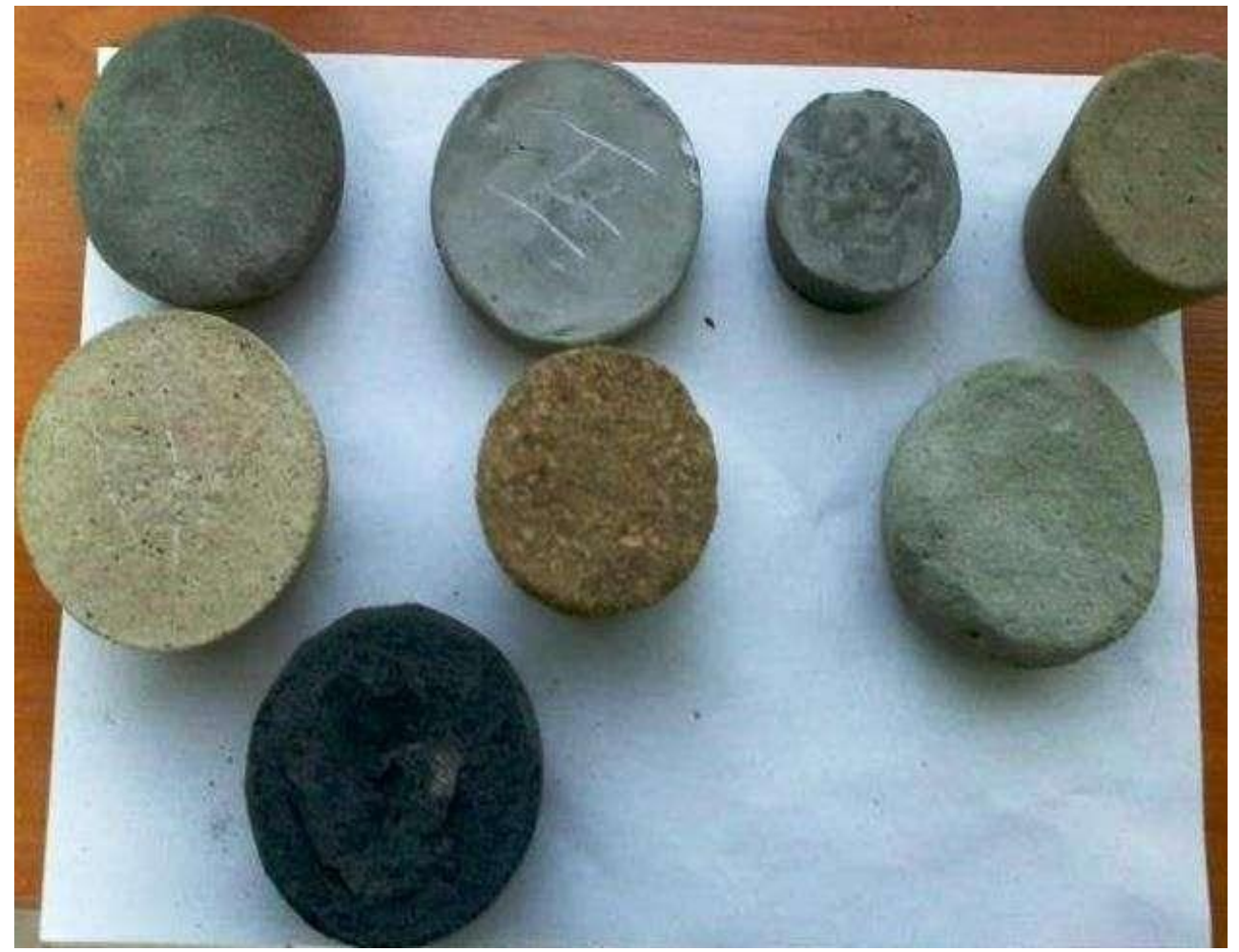

Fig 6:- Image of some of the samples obtained during the experiment. Continuous numbering; in rows, numbering increases from left to right:

\section{1st row:}

1. standard batch of standard grinding gypsum;

2. standard batch of nano-dispersed gypsum;

3. standard batch of standard gypsum grinding; after daily exposure, the sample was impregnated with the orthosilicic acid by dipping into its gel, then after daily exposure to air it was kept under water for 10 days (the sample became waterproof after impregnation);

4. standard batch of a mixture of gypsum and calcium carbonate (grinding rubble stone) with a weight parts ratio of 1:1 nano-dispersed grinding, after daily exposure, the sample was impregnated with the ortho-silicic acid by dipping into its gel, then after daily exposure to air it was kept for 10 days under water (the sample after impregnation also became waterproof);

\section{2nd row:}

1. Standard mixture batch: a) coarse sand, b) calculated mixture of a nano-dispersed sand powder with grinding of stone rubble at a ratio of $4: 1$ and $10 \%$ of this alkali mass 
2. Standard ortho-silicic acid gel-based mixture batch: sand, nano-dispersed grinding of stone rubble at a ratio of 3:1.

3. Standard water-based mixture batch: sand, nano-dispersed grinding of kaolin clay at a ratio of $6: 1$.

$3^{\text {rd }}$ row:

Standard water-based mixture batch: a) nano-dispersed grinding of coal ash with $10 \%$ of its alkali weight; sand at a ratio of 1:4.

Figure 8 illustrates the possibility of obtaining nano-cement from gypsum, rubble, coarse sand, kaolin, and grind coal ash using the technology developed by us. That is, brittle and currently not very popular in the construction industry cheap materials the use of which for these purposes was not previously possible.

\section{Results And Discussion:-} concerning the problem $\mathrm{C}$ :

Below, we will list only some of the UMoID/A applications:

1. The production of low-cost (four times cheaper than existing due to the lack of components sintering stages in production) especially firm, alkaline nanocements resistant to sea water - without clinker burning, using local smelter slags, rocks, and volcanic rocks;

2. Large-scale construction using $3 \mathrm{D}$ printing based on the use of a special liquid glass created by us obtained using cheap nanotechnology without sintering components, which significantly increases the strength of concrete and papercrete , as well as significantly reducing the "setting" process;

3. Improving the efficiency of oil cracking and obtaining cheap drillfluids;

4. Obtaining high-quality homogeneous and poorly settleable matter as effective boiler or furnace oil for thermal power plants and cottages and cheap diesel fuel for use in powerful diesel engines of small power plants or powerful shiplquarry diesel engines (mining dump trucks, tractors, earth movers, excavators, as well as helicopters, transport ships, and harbor tugs);

5. Production of water with reduced content of salts, heavy metals and dissolved gases in the UMoID/A.

6. In the food industry: to obtain cheap compound feeds, supersaturated solutions, caramelized solutions, to obtain molasses directly from starch, without chemical hydrolysis, biological activation of water, as a butter churn and to produce starch [12], margarine and mayonnaise, which is 200 times more effective than ball mills when receiving the suspension.

We personally conducted a large number of grueling tests in difficult conditions using the UMoID/A that we constructed, which showed to groundbreaking results:

1. An industrial method for nano-cement production has been developed. A notice on this invention [14] Eurasian patent № 201800299 has been received.

2. A new nanotechnological industrial method for producing the ortho - silicic acid (liquid glass) has been developed. A notice on this invention [15] Eurasian patent № 201800298 has been received.

\section{Conclusion:-}

\section{on the problems $A$ and $B$ :}

These newly developed nanotechnologies allow to implement the widest range of effective and cheap state projects: from the construction of new cities, ports, coastal and underwater tunnels, underwater objects, tower buildings, multikilometer highways to small cottages, and infrastructure of holiday villages.

In total, we have developed about thirty revolutionary, fundamentally new, cheap, and effective nanotechnologies (more than ten of which can be used in the food industry) based on the use of new nanotechnologies and universal modules of industrial disintegrators/activators (UMoID/A) patented by us. This allows for a several-fold increase in revenues of the corporate groups, corporations, and firms that possess corresponding patents and nanotechnologies and work in nanoenergetics and nanotechnology.

Owners of enterprises receive huge profits from using the devices [9], [10]. - the enterprise's income includes heat and electricity, heated steam, and a solid conglomerate of crystalline formation generated at these enterprises according to the operating technologies proposed. Running an enterprise becomes simpler and cheaper when it comes to manufacture - there is no need for exhaust gas cleaning devices and heat recovery boilers. 
Thus, the problem of creating new nature-like energy technologies that do not harm the outside world but exist in harmony with it and allow us to recover the disturbed balance between the technosphere and the biosphere is solved.

Saving the planet's population from an impending crisis and pandemic is an extremely simple, effective, and economically profitable step - to introduce the

Sixth Technological Revolution nanoenergetics as soon as possible. In addition to the huge economic impact, all exhaust gases, representing a mixture of metal oxides belonging to the middle groups of the D. I. Mendeleev's Periodic Table, closest to a non-ideal plasma, are obtained both during operation of metallurgical, cement, energy, coal enterprises and also by processing and utilization of industrial and municipal waste, ore and energy raw materials processing, slag utilization, metal radioactive waste processing, etc. are a blank for the production of quantum condensate of a non-ideal plasma as an energy source for modern nanoenergetics and then, in accordance with the technology developed by the author, they should be transferred to and processed in the devices proposed by the author [9], [10] for thermal or electric energy generation. Thus, there will be no gases hazardous to human health in the emissions of such enterprises, and people's immunity will get a boost, the purification of planet Earth will begin.

Conclusion on the problem C - Mitigating the effects of the current economic crisis and dealing with the global economic collapse.

The tragedy of the current situation in the world, unfortunately, is not limited to the medical problem of the panepidemic in the world. Modern medicine is forced to recognize that coronavirus will not disappear completely even after the end of the pandemic. Moreover, a number of virologists suggest another (even more dangerous) strain of the virus next year.

The International Monetary Fund announced a global economic recession. The pandemic has already undermined the global economy and exacerbated existing problems of poverty and inequality. There are predictions that we will have to live through a crisis, famine, and social unrest. To avoid such a development of events, it is necessary to invest resources in a survival strategy, that is, in the projects of fundamentally new technologies of the Sixth Technological Revolution which at a minimum cost of technology, materials, and labor costs can generate an enormous source of added value, while providing a huge number of jobs.

Such projects are the latest nanotechnology projects and the immediate financing of these mega-projects is saving the world from hunger, social unrest, crisis with all its consequences, and in this case the following can be said: "Defer No Time, Delays Have Dangerous Ends."

The transition to the new, modern, efficient, renewable, harmless, economically viable (fully recouped) nanoenergetics of the Sixth Technological Revolution - naturally, simultaneously with reforming the waste processing and disposal industry - recycling (comparable in terms of financial volume to the entire transport and energy complex), which should be considered a national project capable of providing jobs for tens of millions of people who have lost small and medium-sized businesses and bring additional revenues to holding companies, corporations, and firms that possess corresponding patents and technologies and are engaged in nanoenergetics and nanotechnologies.

The national (fully recouped) project based on the use of universal industrial modules of disintegrators/activators (UMoID/A) - the devices with a vortex ferromagnetic layer [9], [10], which allow to implement a huge range of cheap and effective national regional projects for the production of new materials and products in the widest technical and financial ranges, providing jobs for tens of millions of people who lost their jobs during the crisis and saving trillions of US dollars of the state budget of each country that managed to adopt ways to mitigate the impact of the current economic crisis and recession, designed and patented by the corresponding members of RAS, PhD in Physics \& Mathematics, Prof. A.V. Kulakov and Candidate of Sciences in Physics and Mathematics V.A. Rantsev - Kartinov.

\section{Conclusion:-}

And, of course, I would like to express my sincere gratitude and deep appreciation to my teachers, outstanding scientists, senior fellows, and colleagues at my previous job who have already passed away, to Academician A.P. Aleksandrov and Academician, Nobel Laureate in physics A.M. Prokhorov, who not only explained to me the scale of what I have discovered in physics and what to do next, but also the peculiarities of my future fate: they told me: "Keep in mind, a person ahead of his time, waiting for its arrival in uncomfortable conditions and remember for life the 
following prophetic words of the great Russian scientist V.I. Vernadsky: "The whole history of science at every step shows that individuals were more right in their statements than entire corporations of scientists or hundreds and thousands of researchers adhering to prevailing views... Undoubtedly, in our time the most true, most correct, and deepest scientific worldview lies among some individual scientists or small groups of researchers whose opinions do not attract our attention or excite our dissatisfaction or denial." These instructions allowed me to withstand the blows of scientific fate and continue my scientific work.

In my heart, a memory will always be kept off my friend, a wonderful scientist, and just a wise man, PhD in Physics \& Mathematics - Rumiantsev A. A.

Finally, I would like to express my heartfelt gratitude to $\mathrm{PhD}$ in Engineering V.M. Tiutiunnik and Candidate of Sciences in Physics and Mathematics V.A. Rantsev- Kartinov for the joint work on universal industrial modules of disintegrators/activators.

\section{References}

[1] A.V. Kulakov, A.A. Rumiantsev. Spontaneous magnetization of plasma of quantum origin, Journal of Technical Physics, 1988, Volume 58, Issue 4, p. 657- 660.

[2] A.V. Kulakov, E.V. Orlenko, A.A. Rumiantsev. Quantum exchange forces in condensed matter, Moscow, Nauka Publishing House, 1990.

[3] A.V. Kulakov, A.A. Rumiantsev. Ball lightning as a quantum condensate, Reports of the Academy of Sciences of USSR, Physics, 1991. Volume 320, No. 5, p. 103-1106.

[4] A.V. Kulakov, V.M. Tiutiunnik. Solid phase plasma ultraviolet laser, International Journal of Advanced Research, 2017, Vol. 5(4), pp.271-273.

[5] A.V. Kulakov, V.A. Rantsev-Kartinov. Experimental confirmation of the existence of plasma quantum condensate, Izvestia RAS. Energetics, 2015, No.1, p. 46-61.

[6] A.V. Kulakov, A.A. Rumiantsev. Generation of high-energy particles by MPD shock turbulence, Journal of Technical Physics, 1979. Volume 49, Issue 10, p. 2127-2132

[7] A.V. Kulakov. Quantum plasma condensate as a new source of electric energy. MHD - generator with plasma separation. International Journal of Advanced Research-2017 - Res. 5(8).2004-2011.

[8] A.V. Kulakov. Quantum non-ideal plasma as a source of heat energy. Plasma fuel combustion International Journal of Current Research. - 2017.-Vol. 9. P.6. P.53361-53365.

[9] V.A. Rantsev-Kartinov, A.V. Kulakov. Universal module of industrial disintegrators/activators, Utility Model Patent No. 161751, Bulletin of the Federal Service for Intellectual Property, Patents, and Trademarks No. 13, dated $05 / 10 / 2016$ 
[10] A.V. Kulakov, V.A. Rantsev-Kartinov. Eurasian patent for invention No. 029979 Device for a universal module of industrial disintegrators/activators June 29, 2018.

[11] P.L. Kapitsa. Why is fame needed?, Priroda Magazine, 1994, No. 4 (944)

[12] A.V. Kulakov, V.A. Rantsev-Kartinov, and V.M. Tiutiunnik. Application of universal multipurpose modules of industrial disintegrator-activators for the processing of cereals and potatoes into starch products International Journal of Advanced Researc.2017 Res 5(5).1759-1762

13 A.V. Kulakov. Quantum plasma condensate. Cold nuclear fusion. New nano- technologies. LAMBERT Academic Publishing. 2019

[14]A.V. Kulakov V.A., Rantsev-Kartinov Eurasian Patent Application № 201800299 for the invention METHOD OF MAKING NANOCEMENTAL. A.

[15].A.V.Kulakov, V.F.Rantsev-Kartinov Eurasian Patent Application №201800298 for invention NEW NANOTECHNOLOGICAL INDASTRIAL METHOD OF PRODUCING ORTHO-SILICIC ACID. 
